\title{
Phase Clocks for Transient Fault Repair
}

\author{
Ted Herman* \\ University of Iowa, Department of Computer Science \\ herman@cs . uiowa. edu
}

15 July 1999, revised 10 July 2000

\begin{abstract}
Phase clocks are synchronization tools that implement a form of logical time in distributed systems. For systems tolerating transient faults by self-repair of damaged data, phase clocks can enable reasoning about the progress of distributed repair procedures. This paper presents a phase clock algorithm suited to the model of transient memory faults in asynchronous systems with $\mathrm{read} /$ write registers. The algorithm is self-stabilizing and guarantees accuracy of phase clocks within $O(k)$ time following an initial state that is $k$-faulty.
\end{abstract}

Index Terms: distributed algorithms, fault tolerance, fault containment, synchronizers, self stabilization, time adaptive

\section{Introduction}

Measuring time is widely recognized as an important system service and greatly simplifies the construction of many distributed algorithms. The reason, simply put, is that deductions about the progress of concurrent activities, made by measuring elapsed time, effectively substitute for communication and protocols that directly monitor such progress. Of course this technique can only be used to the extent that a distributed system is synchronous, matching its progress with elapsed time. Yet so attractive is the use of time to simplify algorithm construction, that even in asynchronous systems, researchers seek to simulate synchrony [4], introduce logical clocks [16], and/or logical time [17] as programming tools.

One illustration of logical time in an asynchronous system is the organization of a computation into phases. The basic property of a phased computation is that a process does not enter phase $(k+1)$ until each related process has completed phase $k$. The case where all processes are related is equivalent to barrier synchronization, and the case where the relation between processes is specified by a graph corresponds to a phase clock. Many implementations of phased computation simply use a counter, called a clock, to represent the current phase number of a process. Consider the graph relation between processes to be a network communication topology, where the graph has diameter $\mathcal{D}$ and distance between processes $p$ and $q$ is denoted by dist $t_{p q}$. A phase clock invariantly relates phase numbers and distance as follows: any process $p$ has $c l o c k_{p}=k+d$ only if $c l o c k_{q} \geq k$ holds for each process $q$ satisfying $d_{i s t} t_{p q}=d$ (notice that $k=1$ is just the basic property mentioned above). Thus

*This work is supported by NSF CAREER award CCR-9733541. 
if clock $_{p}=k+d$, holds at some state, we deduce that clock $_{q}=k$ holds currently or held at some previous state. This is a useful timing property because programs can use phase clocks for inferences about nonlocal information relayed through neighboring processes. For example, process $p$ could use its clock to infer termination of a broadcast operation, rather than use explicit termination detection, by waiting for sufficiently many increments to $\operatorname{clock}_{p}$ (assuming that the broadcast operation is geared to the phase clock).

Phased computation is a reasonable discipline for many activities of a distributed system, including procedures invoked as part of fault diagnosis and repair. The fault domain for this paper is the model of transient faults, which corrupt local process states and communication registers, but do not damage a system's control logic. It is therefore feasible for a system to self-diagnose and restore variables corrupted by a transient fault to values that enable correct system function. One of the difficulties in using phase clocks to control distributed repair activities is that faults may corrupt local clock values. The phase clock protocol presented in this paper not only repairs clock values corrupted by a transient fault, but does so in a manner that enables the system to use the phase clock for other repair activities.

Contributions. This paper presents a distributed phase clock, called the repair timer, specialized for the task of transient fault repair in a distributed system. The repair timer is time adaptive, meaning that it satisfies desired accuracy and progress properties within $O(k)$ time after any transient fault event corrupting $k$ processes. The repair timer differs from standard phase clocks because it starts at zero and halts after repair is complete (behaving somewhat like an egg-timer); this enables direct inspection of elapsed repair time, which standard phase clocks do not provide?. The repair timer is also a self-stabilizing algorithm, able to restore all variables to a legitimate state following any transient fault event or combination of transient failures. Finally, the paper presents composition theorems to show how the repair timer is useful for the timing of fault repair procedures in a distributed system.

Related work. Many recent works are motivated by what is seen as pessimism in the model of selfstabilization, which does not discriminate between cases of severe transient faults and minor transient faults. In addition to the desired robustness of self-stabilization, fast stabilization of output variables has been recently demonstrated in a number of algorithms 11, 9, 5n and some general methods to achieve time adaptivity [14, 15, 8] or local self-stabilization [10, 1].

Self-stabilizing phase clocks are given in 12, 2, 6]. None of these constructions guarantee fast stabilization for cases of limited transient faults, and all appear to require lengthy stabilization time (proportional to the diameter of the communication graph) in some cases where only a single process variable is corrupted by a transient fault. Requirements for a repair timer are described in [13], which is a precursor to this paper.

Contents. Section 2 presents the computation and system model for the paper. Section 3 presents the algorithm for the repair timer and Section 1 verifies the self-stabilization and time adaptive properties of the algorithm. To illustrate the use of the repair timer, Section 5 describes two designs incorporating the repair timer as a component in a system. The paper's concluding remarks are the subject of Section 6. Proofs of technical lemmas have been moved to the paper's Appendix.

\footnotetext{
${ }^{1}$ To see why this is not trivial, suppose some time-adaptive phase clock were available, and consider measuring elapsed repair time by recording the start time of repair in some local variable; such a local variable could, however, have an erroneous value due to a transient fault. Since transient faults do not provide any signal at the start of repair, a process cannot locally decide whether its local variables are accurate or not.
} 


\section{Distributed System}

The system consists of a fixed set of $n$ processes that communicate by reading and writing shared registers. Communication between processes is limited to a network represented by an undirected, connected graph: for any pair of processes $(p, q)$ there exist a pair ( Register $_{p q}$, Register $_{q p}$ ) if and only if there is an edge between $p$ and $q$ in the communication graph. Process $p$ is the only writer of $\operatorname{Register}_{p q}$ and $q$ is the only reader of Register $p q$. A process cannot read the registers it writes. Registers thus approximate message passing with bounded buffers, and a self-stabilizing simulation of link registers using messages is described in [7]. A register can have numerous fields used to write values of different local variables (just as numerous local values can be transmitted in fields of one message).

If $(p, q)$ is an edge in the communication graph, then $p$ and $q$ are called neighbors, which is denoted by $p \in \mathcal{N}_{q}$ or equivalently, $q \in \mathcal{N}_{p}$. The diameter of the communication graph is $\mathcal{D}$. The distance between any pair $(p, q)$ in the graph is denoted by dist $t_{p q}$. The term region refers to a connected component of the graph that has some property of interest.

Each process is an autonomous, finite-state computing entity. We use conventional imperative programming notation and concepts to describe the operation of a process, so each process has a program counter and program statements that manipulate variables. A subset of these variables are called output variables, which directly support the system's intended function.

A configuration of $p$ is a specification of values, one for each of process $p$ 's variables, the value of $p$ 's program counter, and a value for each register that $p$ writes. A (system) state is a vector of process configurations, one configuration for each process in the system. Any function from the set of all states to the set $\{$ true, false $\}$ is called a state predicate.

A process step is either a register operation (and corresponding advancement of the program counter) or some modification of internal and output variables (and program counter) of that process. A computation is an infinite sequence of states so that each consecutive pair of states corresponds to a process step and the sequence of states includes an infinite number of steps of each process. We thus assume that computations are fair; more precisely, we assume weak fairness in that no process is prevented from executing steps in a computation. We use the term computation segment to denote a finite, contiguous subsequence of a computation.

The program of each process specifies a cycle, which consists of three parts: $(i)$ a process reads the registers written by each of its neighbors, $(i i)$ the process possibly assigns values to its variables, and (iii) the process writes registers for each of its neighbors. The definition of a cycle is a convenient and simple abstraction for measuring the progress of a process in a computation.

The system is designed to accomplish some task represented by a state predicate $\mathcal{L}_{O}$. Whether or not $\mathcal{L}_{O}$ holds at a given state is solely determined by the values of output variables. A predicate $\mathcal{L}$ is called a legitimacy predicate iff $\mathcal{L}$ is a system invariant and $\mathcal{L} \Rightarrow \mathcal{L}_{O}$. A state $\sigma$ is output-legitimate if $\mathcal{L}_{O}$ holds at $\sigma$, and is legitimate if $\mathcal{L}$ holds at $\sigma$. It is often preferable to specify legitimacy (or output legitimacy) in terms of the behavior of processes rather than explicitly specifying a state predicate. A formal definition of legitimacy in terms of behaviors is possible, but to streamline the presentation, the state-based definition is used in this paper. Where process behavior is important in this paper, we verify separately that the system exhibits the desired behavior.

Because each iteration of a process program specifies a cycle, time is conveniently measured in asynchronous rounds, which are defined inductively. A round of a computation, with respect to an initial state $\sigma$, is a computation segment originating with $\sigma$ of minimum length containing at least one complete cycle (from reading registers to writing registers) of each process. The first round of a computation consists of a round with respect to the initial state of the computation, and round $k$ of a computation, $k>1$, consists of a round with respect to the first state following round $k-1$. 
A round is, roughly speaking, one unit of "parallel time" in the system. A notion similar to a round is commonly used to analyze the complexity of message-passing protocols by normalizing message delay to the maximum message delay [3]. For analysis in this paper, the notion of a round is further refined. An $\mathrm{R}_{p}^{d}$-round starting from a state $\sigma$ is a computation segment of minimal length containing at least one complete cycle of each process in the set $\left\{q \mid\right.$ dist $\left._{p q} \leq d\right\}$. A round is thus equivalent to an $\mathrm{R}_{p}^{\mathcal{D}}$-round for any choice of $p$.

A system is self-stabilizing if every computation contains a legitimate state (that is, for any initial state, the system eventually reaches a legitimate state). The stabilization time is the worst case number of rounds in the prefix of a computation that does not contain a legitimate state. Proving that a system is self-stabilizing entails demonstrating that a predicate $\mathcal{L}$ is invariant, implies $\mathcal{L}_{O}$, and that every computation contains some state satisfying $\mathcal{L}$.

A fault event is a non-computational operation that modifies variables, program counters, and/or registers. More formally, a fault event can be any pair of states (whereas a consecutive pair of states in a computation is a process step). Computations do not include fault events; a system history could be a sequence of states consisting of computation segments punctuated by fault events. Reasoning about fault repair proceeds with respect to each computation segment, since the system cannot anticipate whether or not another fault will occur.

A state $\sigma$ is $k$-faulty if $k$ is the minimum number of process configurations in $\sigma$ that, if appropriately changed, transform $\sigma$ into a legitimate state. The number $k$ thus corresponds to the Hamming distance from $\sigma$ to the nearest legitimate state. There may be numerous ways to transform $\sigma$ to a legitimate state by changing $k$ process configurations, some in which process $p$ 's configuration changes, and others where the transformation does not change $p$ 's configuration. It is convenient to resolve this ambiguity by some unique, deterministic choice of which processes should change configurations to obtain a legitimate state from $k$-faulty state $\sigma$. With such a deterministic choice, process configurations of $\sigma$ can be labelled faulty or nonfaulty depending on whether they should change or not. This deterministic choice can further be refined to label variables and register fields as either faulty or nonfaulty. How such a deterministic choice should be implemented turns out not to be an issue in the sequel; for the repair timer given in Section 3 there is an unambiguous definition of a faulty process configuration and for the interface proposed in Section 5 it is only required that if a faulty process configuration neighbors a nonfaulty process configuration, then the presence of a fault can be detected (which for many systems is the case even by reversing the choice of which of these two neighboring configurations is considered to be faulty).

The main emphasis of this paper is time-adaptive, stable repair of output variables, meaning that a system should stabilize its output variables to satisfy $\mathcal{L}_{O}$ from any $k$-faulty initial state after at most $O(k)$ rounds. Formally, a system is time adaptive if each computation starting from any $k$-faulty initial state $\sigma$ contains an output-legitimate state $\sigma^{\prime}$, within $O(k)$ rounds following $\sigma$, such that every state following $\sigma^{\prime}$ in the computation is output-legitimate. Given this emphasis, it is convenient to extend the terminology for faults: a process $p$ is faulty (nonfaulty) in a computation iff $p$ 's configuration is faulty (nonfaulty) at the initial state.

\section{Algorithm}

One of the difficulties in using phase clocks to control distributed repair activities is that faults may corrupt local clock values. Indeed, repair of the clocks values is a primary concern of this paper, and the usual timing properties of phase clocks must be modified to cope with faults. Two goals for such modifications are: $(a)$ clock values of processes not affected by faults can reliably be used for 
inferences about nonlocal information; $(b)$ the response effort of the system is proportional to the scope of the fault.

Goal (a) seems relatively simple to satisfy, since the clocks of nonfaulty processes have predictable values. However for a standard phase clock, there are ambiguous cases of faulty situations. Suppose neighboring clocks have values $x$ and $x-2$ and only one of these two is a faulty value; there is no obvious way of distinguishing which of these two is faulty. The approach taken in this paper is to use a specialized phase clock for fault repair called a timer. Whereas phase clocks advance throughout system computation, the timer stops advancing when repair is complete. Thus each timer clock reaches a prescribed value $\mathcal{T}$ when the system state is fully repaired. If neighboring clocks have values $\mathcal{T}$ and $\mathcal{T}-2$, then we may conclude that the value $\mathcal{T}-2$ is due to a fault.

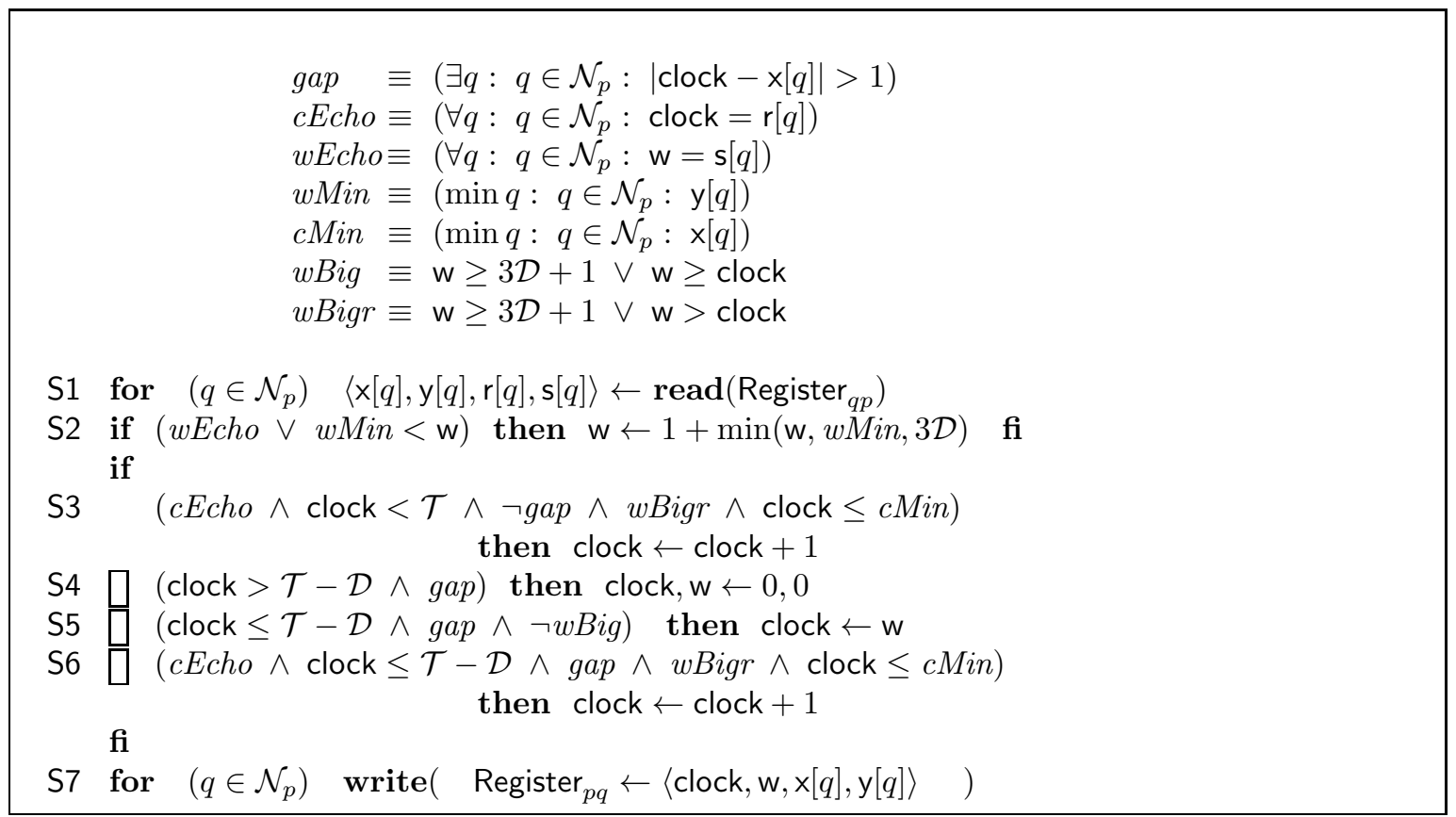

Figure 1: timer for process $p$

Variable Conventions. The variables appearing in Figure f are local variables of process $p$. A number of proof arguments are statements relating variables of different processes, and subscripts are used to distinguish variable ownership (for instance, clock $q$ is owned by $q$ ). Similarly, the predicates defined in Figure 1 are subscripted in definitions and proof arguments (such as $g a p_{p}$ for process $p$ ).

Statement S1 copies four register fields to four local variables, $\langle x, y, r, s\rangle$. Call these variables the image variables. Implicitly the code of Figure 1 defines a mapping from each image variable to a register field and a corresponding "base" variable of a neighboring process (written by statement S7). We say that each image variable is based on a variable of a neighboring process, meaning that the value of an image variable is copied (via register communication) from the variable upon which it is based. Variable $\mathbf{x}_{p}[q]$, for example, is based on clock $_{q}$. Register fields are also images that are statically based on variables.

The meaning of time adaptivity described in Section 2 depends on declaring some of the process variables to be output variables. For the repair timer, let clock ${ }_{p}$ be the output variable of process $p$. 
The output correctness for clock variables is the subject of Section 4.2 .

Program Conventions. The statements S1-S7 given in Figure 1 describe one complete cycle of the repair timer for process $p$. Therefore, after executing S7, process $p$ executes S1 to start the next cycle. The group of statements S3-S6 constitute a multiway if statement; in any cycle, at most one of S3-S6 are executed.

Statements S2-S6 specify internal calculations for process $p$, since they manipulate local variables. In a computation, we suppose that each of these statements specifies one computation step. Statements S1 and S7 specify each $\left|\mathcal{N}_{p}\right|$ computation steps, since a step can read or write at most one register. Ordering of the read and write operations of S1 and S7 is unimportant to the algorithm.

Algorithm Structure. To understand the algorithm of Figure 1 it is useful to first ignore statements S3-S6 and focus attention on the $\mathrm{w}$ variables. Notice that statement S2 will reduce $\mathrm{w}_{p}$ if any of the registers read by $\mathrm{S} 1$ imply a value $\mathrm{w}_{p}-2$ or smaller for any neighboring $\mathrm{w}$ variable. The global effect of many processes executing S2 can thus be "convergence to the least w" over a number of rounds. The result of executions of S2 will, in general, lead to a situation where neighboring process $\mathrm{w}$ variables differ by at most 1 , which is one of the properties of a phase clock. The wEcho condition of S2 allows any process with a globally minimal $w$ variable to increment its $w$ variable after all neighbors acknowledge its current value, via the s image variables (which occurs within two rounds). Therefore the set of $w$ variables apparently enjoy both properties of a phase clock - that neighboring $w$ variables differ by at most 1 and increase continually (until the upper bound of $3 \mathcal{D}+1$ occurs) in a computation.

Why not simply use the $\mathrm{w}$ variables for repair timing and dispense with the logic of S3-S6? The answer lies in the additional constraint we impose for faulty initial states. For repair purposes, it is not enough for clocks to be in phase and increment, they should also be accurate, meaning that the value of a clock should be a measure of how long computation has progressed after the initial detection of a fault. The $w$ variables do not have this property. For instance, a faulty initial state could have $\mathcal{D}$ as the initially smallest $\mathrm{w}$ variable, so that all subsequent states have $w$ variables overstating the repair time at least by $\mathcal{D}$. An attempt to fix this problem would be some statement similar to S4, that would reset $w$ to zero whenever neighboring $w$ variables differ by more than 1 . It is easy to construct examples of computations where such an attempted fix will fail because $w$ variables are reset to zero infinitely often. This kind of idea can work, however, if any w variable were guaranteed to be reset to zero at most once in a computation, and that is the basic idea behind statements S3-S6, which reset a clock variable to zero at most once in a computation. Although $w$ variables do not enjoy the accuracy needed for repair timing, they provide a useful "reset layer" for the clock adjustments of the algorithm.

Definition 1 A state is timer-final if every register field and image variable value is equal to the value of its base variable, and $\left(\forall p::\right.$ clock $\left._{p}=\mathcal{T} \wedge \mathrm{w}_{p}=3 \mathcal{D}+1\right)$. A process configuration is timer-final if all its variables and register fields have values corresponding to a timer-final state. We define predicate $\mathcal{L}_{T}$ to hold for a state iff that state is timer-final.

The value $\mathcal{T}$ used in the algorithm and Definition 1 is a constant adequate for the fault tolerance of the repair timer and for the application of the timer, as discussed in Section 5 . The proof of self-stabilization of the repair timer requires only that $\mathcal{T} \geq 11 \mathcal{D}$. 
Verification. The verification of desired repair timer properties is divided into two stages. First, the algorithm is considered as an isolated component, so that faulty states are those states deviating from Definition 11. Section 4.1 is devoted to a proof that the repair timer self-stabilizes to a timer-final state. Section 4.2 presents the proofs that apply to $k$-faulty initial states, showing that the repair timer achieves desired accuracy after $O(k)$ time following the initial state.

The second stage of verification is concerned with integration of the repair timer as a component of a system. The timer is a tool for time adaptive repair. Discussion of how the timer is used is deferred to Section 5 , where it is explained that the timer is a service with only one operation, namely to start the timer by assigning clock $\leftarrow 0$; thereafter, the clock should increment as a phase clock. Although a system state's legitimacy depends on variables of all system components, the simple interface between the timer and other system components makes it reasonable to consider fault tolerance properties of the timer in isolation, which motivates the two stage approach to verification.

\section{Stabilization and Adaptivity}

\subsection{Self-Stabilization}

Each process writes its communication registers in every cycle from its variables. Therefore, following the first round of any computation, all register fields are equal to current or previous values of the corresponding base variables. Following the second round, each image variable has a value previously written from the corresponding base variable. Moreover, following the third round of a computation, the third and fourth fields of Register ${ }_{q p}$ contain values previously written by $p$ and then copied by $q$. It is convenient to assume that register fields correspond to values previously written in the computation, so we call a computation based if it is the suffix, starting from round three or higher, of another computation.

Statements S4 and S5 have the only assignments that may reduce the value of clock variables. We call a computation (or computation segment) reset-free if no process executes S4 or S5 in that computation. A computation is called rising if it is the suffix of a based, reset-free computation such that each process has read its registers at least once in the based, reset-free computation prior to the first state of the suffix. Rising computations enjoy the useful property that at all states, the value contained in $x_{p}[q]$ is a lower bound on the current value of $\operatorname{clock}_{q}$. (This property follows because the computation is resetfree and each process previously read registers and assigned to its $\mathrm{x}$ variables while the computation was reset-free.)

\section{Definition 2}

$$
b_{p q} \equiv\left(p \notin \mathcal{N}_{q}\right) \vee\left(\left|\operatorname{clock}_{p}-\operatorname{clock}_{q}\right|<2 \wedge \mathrm{x}_{p}[q] \leq \operatorname{clock}_{q} \wedge\left|\operatorname{clock}_{p}-\mathrm{x}_{p}[q]\right|<2\right)
$$

A state is smooth if $\left(\forall p, q:: b_{p q}\right)$. A set of processes $P$ forms a smooth region if the subgraph of the communication topology induced by $P$ is connected and $\left(\forall p, q: p, q \in P: b_{p q}\right)$.

Lemma 1 In a rising computation, $\left(b_{p q} \wedge b_{q p}\right)$ is an invariant for any pair of processes $p$ and $q$.

Lemma 2 Let $\sigma$ be the first state of a rising computation segment such that for $p \in \mathcal{N}_{q}$, both clock and clock $_{q}$ have incremented at least once in the computation segment. Then $\left(b_{p q} \wedge b_{q p}\right)$ holds at state $\sigma$. 
Lemma 3 If each clock variable has incremented at least once prior to state $\sigma$ in a rising computation segment, then $\sigma$ is smooth.

Lemma 4 Smoothness is invariant for a based computation; within $O(\mathcal{D})$ rounds following a smooth state, a based computation contains a timer-final state.

Lemma 5 If clock $k_{p}$ is less than $\mathcal{T}$ and less than or equal to all neighboring clock values at the initial state of a based, reset-free computation segment, and $\mathrm{w}_{p}=3 \mathcal{D}+1$ holds at the initial state, and

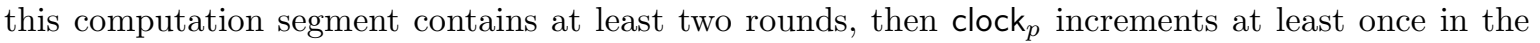
computation segment.

Lemma 6 Let the initial state of a based computation satisfy $\left(\forall p::\right.$ clock $\left._{p} \leq 7 \mathcal{D} \wedge \mathrm{w}_{p}=3 \mathcal{D}+1\right)$. The computation contains a state where $\left(\exists q:: \operatorname{clock}_{q}=10 \mathcal{D}+1\right)$; the first state satisfying $(\exists q::$ clock $\left._{q}=10 \mathcal{D}+1\right)$ is a smooth state.

Lemma 7 Let the initial state of a based computation satisfy $\left(\forall p:: \operatorname{clock}_{p} \leq 7 \mathcal{D} \wedge \mathrm{w}_{p}=3 \mathcal{D}+1\right)$. Within $O(\mathcal{D})$ rounds, the computation contains a smooth state.

Lemma 8 Consider a based computation such that $\left(\right.$ clock $\left._{r}=0 \wedge \mathrm{w}_{r}=0\right)$ holds for some process $r$ in the initial state. Within $\mathcal{D}$ rounds there is a state satisfying $\left(\forall p:: \operatorname{clock}_{p} \leq 3 \mathcal{D} \wedge \mathrm{w}_{p} \leq 3 \mathcal{D}\right)$.

Lemma 9 Consider a based computation such that ( clock $_{r}=0 \wedge \mathrm{w}_{r}=0$ ) holds for some process $r$ in the initial state. Within $O(\mathcal{D})$ rounds there is a smooth state or there is a state satisfying $\left(\forall p::\right.$ clock $\left._{p} \leq 7 \mathcal{D} \wedge \mathrm{w}_{p}=3 \mathcal{D}+1\right)$.

Theorem 1 The timer stabilizes to a timer-final state (satisfying $\mathcal{L}_{T}$ ) in $O(\mathcal{D})$ rounds.

Proof: The invariance of $\mathcal{L}_{T}$ is verified by observing that none of S1-S6 change any variable value at a timer-final state. Convergence is demonstrated by a sequence of claims about an arbitrary computation $A$. Let $B$ be a suffix of $A$ beginning following the second round of $A$; by definition, $B$ is a based computation. We consider two cases for $B$.

Case: $B$ contains no step executing S4 within $O(\mathcal{D})$ rounds. By arguments similar to those given in the proof of Lemma 9, some state of $B$ satisfies $\left(\forall p:: w_{p}=3 \mathcal{D}+1\right)$ within $O(\mathcal{D})$ rounds and continues to hold at least until S4 executes. Let $C$ be a suffix of $B$ satisfying $\left(\forall p:: w_{p}=3 \mathcal{D}+1\right)$ at its initial state. Observe that $C$ is based and reset-free for $O(\mathcal{D})$ rounds, so Lemma 5 is applicable to $C$. Within $O(2 \mathcal{T})$ rounds of $C,\left(\forall p::\right.$ clock $\left._{p}=\mathcal{T}\right)$ holds, and the state satisfies $\mathcal{L}_{T}$.

Case: $B$ contains some step executing $\mathrm{S} 4$ within $O(\mathcal{D})$ rounds. Execution of $\mathrm{S} 4$ results in a state satisfying the premise of Lemma 9. Therefore $B$ either contains a smooth state within $O(\mathcal{D})$ rounds, or contains a state satisfying $\left(\forall p::\right.$ clock $\left._{p} \leq 7 \mathcal{D} \wedge \mathrm{w}_{p}=3 \mathcal{D}+1\right)$ within $O(\mathcal{D})$ rounds. The latter possibility is the premise for Lemma 7, which shows that a smooth state is subsequently obtained within an additional $O(\mathcal{D})$ rounds, so with either possibility, $B$ contains a smooth state within $O(\mathcal{D})$ rounds. Lemma 3 implies that $B$ contains a timer-final state within $O(\mathcal{D})$ rounds following a smooth state. 


\subsection{Time Adaptivity}

The desired fault tolerance of the timer consists, informally, of the following two properties. (1) Within $k$ rounds from a $k$-faulty initial state, every clock is accurate, that is, if clock $_{p}=t$ for $t<\mathcal{T}$, it should be that $p$ has incremented clock $_{p}$ as a phase clock $t$ times during the repair procedure. (2) Each faulty process clock is reset to zero and subsequently increments as a phase clock, incrementing to $k$ within $O(k)$ rounds.

Property (1) provides the accuracy needed so that a process can safely wait for distant information to be reliable. Property $(\mathbf{2})$ assures that such distant information arrives in a timely fashion. Because faults may damage clock and other timer variables, Theorem 2 below provides a conditional form of (1), necessarily relaxed to accommodate unusual initial states. Also, some unusual cases of initial states require a conditional form of $(\mathbf{2})$, provided by Theorem 3 .

A system state is faulty if it does not satisfy the definition of legitimacy. In considering the timer in isolation, a state is $k$-faulty if no fewer than $k$ process configurations require change to obtain a timer-final state. However, a complete definition of system legitimacy depends on components other than the timer, so a limited notion of fault is appropriate for the timer.

Definition 3 A set of processes $P$ is unperturbed at state $\sigma$ if $P$ forms a smooth region, $(\forall p: p \in$ $\left.P: \operatorname{clock}_{p}>\mathcal{T}-\mathcal{D}\right)$, and $\left(\forall p, q: p \in P \wedge q \in \mathcal{N}_{p} \wedge q \notin P:\right.$ clock $\left._{p}=\mathcal{T} \wedge \mathrm{x}_{p}[q] \geq \mathcal{T}-1\right)$. A process $p$ is unperturbed at $\sigma$ if there exists an unperturbed region containing $p$; process $p$ is perturbed if there exists no unperturbed region containing $p$. State $\sigma$ is $k$-perturbed iff $k$ is the number of perturbed processes at $\sigma$.

The motivation for this definition derives from the ambiguity of certain clock values and nondeterminism of asynchronous computation. Some proofs are simplified using Definition 3, which defines a perturbed process to be a weakening of a faulty process configuration (a nonfaulty process configuration is unperturbed, but the converse may not hold). It follows that if the timer algorithm satisfies desired properties (1)-(2) within $k$ rounds from any $k$-perturbed state, then similar properties also hold for any $k$-faulty initial state. Definition 3 is not useful if $k=0$, so in the sequel any reference to $k$-perturbed state is assumed to imply $k>0$.

Definition 4 Within a computation, a variable clock ${ }_{p}$ is $d$-accurate at a state $\sigma$ if clock $_{p}>\mathcal{T}-\mathcal{D}$ holds, or if clock $p \leq \mathcal{T}-\mathcal{D}$ implies, for $0 \leq m \leq \mathcal{D}$, that the number of $\mathrm{R}_{p}^{m}$-rounds completed prior to

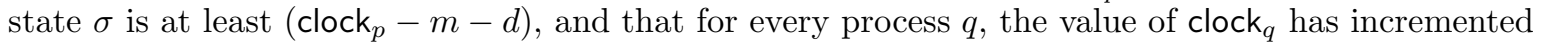
at least $\left(\right.$ clock $_{p}-$ dist $\left._{p q}-d\right)$ times prior to state $\sigma$ in the computation. For a computation initiating from a $k$-perturbed state, a state $\sigma$ is time-accurate if for unperturbed $p$, clock is $d$-accurate for $d=2 \cdot \min (k, \mathcal{D})$, and for perturbed $p, \operatorname{clock}_{p}$ is $d$-accurate for $d=5 \cdot \min (k, \mathcal{D})$.

Definition 4 falls short of the desired precision of property (1), but satisfies safety concerns for many situation of repair timing because a $d$-accurate clock provides a lower bound on the number of cycles that distant processes have completed during repair. For instance, a repair application could depend on a distributed procedure that terminates after $m$ clock increments in a non-faulty environment; this application could wait for $d+m$ clock increments if the repair timer ensures only $d$-accurate clock variables. Unfortunately, an initially faulty state can have arbitrary values in faulty process clock variables, making it impossible to instantly have time accuracy. Theorem 2 given at the end of this section states that time accuracy is guaranteed from any $k$-faulty initial state, provided $k<n$, after at $\operatorname{most} \min (k, \mathcal{D})$ rounds of computation.

Lemma 10 Any process $p$ executes S4, resetting clock ${ }_{p}$ and $\mathrm{w}_{p}$, at most once in any computation. 
Lemma 10 is a corollary of arguments given in the proofs of Lemmas 8 and 9 . It is useful to know that processes execute S4 at most once because any reset step subsequent to S4 is therefore due to S5. Arguments in the proof of Lemma 9 show that $w$ values increase if S4 does not execute, and this idea can be used to establish the eventual increase of clock values.

Lemma 11 Let $\sigma$ be a result of $p$ executing S4. Then for any process $q$ satisfying dist $_{p q}=t \leq$ $\min (k, \mathcal{D})$ there occurs a state $\sigma^{\prime}$, within $t$ rounds following $\sigma$, such that clock $_{q} \leq 3 t \wedge \mathrm{w}_{q} \leq 3 t$; and if there is a path consisting of unperturbed processes from $p$ to $q$, then a state $\sigma^{\prime \prime}$ occurs within $t$ rounds following $\sigma$ such that $\operatorname{clock}_{q} \leq t \wedge \mathrm{w}_{q} \leq t$.

Lemma 11 considers a level of detail not discussed in the proof of Lemma 8 , which supposes based computations. Lemma 11 can also be extended to distances beyond $k$, shown in the following.

Lemma 12 In any computation starting from a $k$-perturbed initial state, for each unperturbed process $p$ satisfying dist $_{p q}=t$ with respect to some perturbed process $q$, the following holds: process $p$ executes $\mathrm{S} 4$ within $4+\min (\mathcal{D}, k+t)$ rounds.

Lemma 13 In any computation beginning from a $k$-perturbed state, any process $p$ satisfying dist $_{p q}=t$ from some perturbed process $q$ does not execute S4 after round $4+\min (2 \mathcal{D}, t+2 k)$.

Lemma 14 Let $\sigma$ be a result of $p$ executing S4. Then for any process $q$, within $t$ rounds following $\sigma$ there occurs a state $\sigma^{\prime}$ such that $\mathrm{w}_{q} \geq \min \left(\left\lfloor\left(t-\right.\right.\right.$ dist $\left.\left.\left._{p q}\right) / 2\right\rfloor, 3 \mathcal{D}+1\right)$ is invariant for the computation beginning with $\sigma^{\prime}$.

Lemma 15 Let $\sigma$ be a result of $p$ executing S4. Then for any process $q$, within $t+2$ rounds following $\sigma$ there occurs a state satisfying $\operatorname{clock}_{q} \geq \min \left(\left\lfloor\left((t-2)-\right.\right.\right.$ dist $\left.\left.\left._{p q}\right) / 2\right\rfloor, \mathcal{T}\right)$.

Theorem 2 Any computation starting from a $k$-perturbed initial state, $k<n$, contains a timeaccurate state $\sigma$ after at most $\min (k, \mathcal{D})$ rounds following the initial state, and all states following $\sigma$ are time-accurate states.

Proof: Provided $k<n$, arguments in the proof of Lemma 12 show that for each perturbed region $R$, some process $r$ executes S4 within the first round, where $r$ satisfies either $r \in R$ or $r \in \mathcal{N}_{q}$ for some $q \in R$. Lemma 11 then implies that within $\min (k, \mathcal{D})$ additional rounds, each $p \in R$ satisfies clock $_{p} \leq 3 \cdot \min (k, \mathcal{D})$. Each unperturbed process clock variable remains larger than $\mathcal{T}-\mathcal{D}$ until S4 is executed, which resets the clock to zero. Thus within $\min (k, \mathcal{D})$ rounds, each clock ${ }_{p}$ is either larger than $\mathcal{T}-\mathcal{D}$ or is at most $3 \cdot \min (k, \mathcal{D})$. After $\min (k, \mathcal{D})$ rounds, unperturbed processes can decrease clock variables to zero, but such a decrease does not falsify the conditions for a time-accurate state.

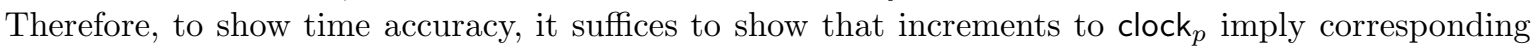
increments have executed at distant processes.

After a process $p$ executes S4, it does not increment clock until Echo holds. If an unperturbed $q$ is a neighbor of $p$, then $p$ does not increment clock $_{p}$ until $q$ has reset clock ${ }_{q}$ and updated the image variables and register fields so that $p$ observes $c E c h o$. It is a simple induction to show that clock cannot increase to a value $t$ unless $q$ has incremented clock $_{q}$ at least $(t-1)$ times. Now consider a minimum length path $P$ of processes, of length $d$, from $p$ to some process $r$, such that each process in 


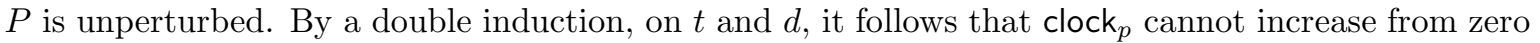
to $t$ unless each process $q \in P$ has incremented clock $_{q}$ at least $t$-dist $t_{p q}$ times. The same argument shows that processes of $P$ complete at least the same number of $\mathrm{R}_{p}^{d}$-rounds in the period where clock increases from zero to $t$.

Returning to the event of $p$ executing S4, we now consider the case of perturbed $q \in \mathcal{N}_{p}$. As observed in the proof of Lemma 11, it is possible that $p$ can increment clock $p_{p}$ twice before $q$ completes a cycle because corrupt values in the initial state enable the $c E c h o$ and wEcho conditions. Furthermore, $p$ can increment clock $\mathrm{k}_{p}$ a third time before $q$ increments its clock because $q$ completes a cycle to enable $c E c h o_{p}$. However in the case of such a third successive increment by $p$, clock $p>$ clock $_{q}$ and $b_{p q} \wedge b_{q p}$ hold as a consequence. Thereafter, we reason about the interaction between $p$ and $q$ as for unperturbed neighbors (note that any subsequent executions of S5 by $p$ or $q$ validate this argument, since we reason about the highest value attained for clock variables after $p$ 's initial three increments). Therefore, the value of clock $\mathrm{k}_{p}$ does not increase to $t$ unless $q$ has incremented clock $q$ at least $t-3$ times. Again, we may consider a minimum length path $P$ of processes, of length $d$, from $p$ to some process $r$, such that each process in $P$ is perturbed (with the possible exception of $p$ ). By a double induction, on $t$ and $d$, it follows that clock $p$ cannot increase from zero to $t$ unless each process $q \in P$ has incremented clock $_{q}$ at least $t-3 \cdot$ dist $_{p q}$ times. Similar arguments show the completion of the appropriate number of $\mathrm{R}_{p}^{d}$-rounds while clock $p$ increases from zero to $t$.

Notice that in the case of a perturbed path of processes, accuracy can diminish by two extra clock units per unit of distance, whereas in the case of an unperturbed path, accuracy corresponds precisely to distance. These observations combined can be used to verify that in any minimum length path $P$ from $p$ to $r$, after $p$ executes S4, the value of clock $_{p}$ increases to $t$ only if for each $q \in P$, the value of clock $_{q}$ has incremented at least $t-$ dist $_{p q}-2 m$ times, where $m$ is the number of perturbed processes in the subpath of $P$ from $p$ to $q$. Since $m \leq \min (k, \mathcal{D})$, time accuracy is verified for $p$.

The arguments above show that time accuracy holds for all unperturbed processes within $\min (k, \mathcal{D})$ rounds and that any subsequent state is $2 \cdot \min (k, \mathcal{D})$-accurate for unperturbed processes. For perturbed processes, similar reasoning applies. Instead of relying on S4 to establish the baseline clock value, we use instead a value bound by the construction given in Lemma 11's proof. Within $\min (k, \mathcal{D})$ rounds, there is a state $\sigma^{\prime}$ where perturbed $p$ has a clock value of at most $3 j$, and $j<\min (k, \mathcal{D})$ is the distance to some unperturbed process that executes S4 in the first round. The value of clock cannot increase from $3 j$ to $3 j+t$ unless process $q$ has incremented its clock at least $t-d_{i s t} t_{p q}-2 m$ times, where $m$ is at most $\min (k, \mathcal{D})$. Therefore when clock $_{p}=x$ at some state following $\sigma^{\prime}$, we infer that clock $_{q}$ has incremented at least $x-3 \cdot \min (k, \mathcal{D})-$ dist $_{p q}-2 \cdot \min (k, \mathcal{D})$ times, which verifies time accuracy for unperturbed processes.

Theorem 2 addresses desired property (1) set out at the beginning of the section. Property (2) specifies that each faulty process clock be reset to zero and then advance as a phase clock. For the same reason that (1) has been weakened to the time accuracy of Definition 4 , we weaken $(\mathbf{2})$ to require only that each perturbed process be reset to some value in the range $[0,3 \cdot \min (k, \mathcal{D})]$ within $k$ rounds following the $k$-faulty initial state, and thereafter increments as a phase clock. Theorem 2 implies that subsequent increases to clock values satisfy a distance property relating the value of a clock to the number of increments of other clock variables. The following theorem states the weakened form of $(\mathbf{2})$.

Theorem 3 For any computation starting from a $k$-faulty initial state, $k<n$, each perturbed process clock is at most $3 \cdot \min (k, \mathcal{D})$ within $\min (k, \mathcal{D})$ rounds and increases to value $\lfloor((t-4)-$ $\min (k, \mathcal{D})) / 2\rfloor$ within $t$ rounds; and each unperturbed process clock similarly increases to $\lfloor(t-4) / 2\rfloor$ within $t$ rounds after resetting by $\mathrm{S} 4$. 
Proof: Lemma 11 directly shows that perturbed processes assign clock variables to at most 3 . $\min (k, \mathcal{D})$ within the first $\min (k, \mathcal{D})$ rounds. Lemma 15 establishes that $p$ increases its clock to at least $m=\min \left(\left\lfloor\left((t-2)-\right.\right.\right.$ dist $\left.\left._{p q}\right) / 2\right\rfloor$ after $t+2$ rounds following the execution of S4. Lemma 12 establishes that for each perturbed region, some process $p$ either within or neighboring the perturbed region executes S4 in the first round. Lemma 15 establishes that processes within a given distance increase their clock values as clock ${ }_{p}$ increases. Any process $q$ within a perturbed region containing or neighboring $p$ is at most distance $\min (k, \mathcal{D})$ from $p$; simplifying the bound of Lemma 15 using $\min (k, \mathcal{D})$ as a distance upper bound yields a lower bound of $\operatorname{clock}_{q} \geq\lfloor((t-2)-\min (k, \mathcal{D})) / 2\rfloor$ after $t+2$ rounds.

\section{Embedded Timer}

This section discusses use of the repair timer as a component in a system. Whereas Section 1 investigated properties of the repair timer in isolation, the results of this section are essentially composition theorems stating conditions under which the repair timer can be used as a tool to enable time-adaptive fault tolerance in a system.

Consider a system that uses the repair timer as one of its components. The term core system is used in this section to refer to all system components outside the repair timer; in other words, the entire system consists of the core system plus the repair timer. The elements of a process configuration (variables and registers) can be partitioned into those belonging to the repair timer and those belonging to the core system. The timer projection of a state is formed by removing all elements from each process configuration not relevant to the repair timer (that is, only clock, w, related image variables and register fields are retained). A core projection is formed by removing all repair timer elements from the state.

Requirement 1 Output legitimacy $\mathcal{L}_{O}$ of the system is defined solely in terms of the core projection, that is, no repair timer variable is an output variable. Core system legitimacy, given by the predicate $\mathcal{L}_{C}$, is also defined with respect to the core projection; predicate $\mathcal{L}_{C}$ is independent of repair timer variables or register fields. The legitimacy predicate for the system is $\mathcal{L} \equiv \mathcal{L}_{C} \wedge \mathcal{L}_{T}$.

The interface between core system and repair timer is illustrated in Figure 2. Communication between these two components occurs in each process, but is restricted to two methods: the core system can reset the clock and $w$ variables, and the core system may read the current clock value. Henceforth the term double-reset is used to denote the assignment clock, $w \leftarrow 0,0$. Both S4's assignment of Figure 1 and the core system's assignment illustrated in Figure 2 are double-reset assignments.

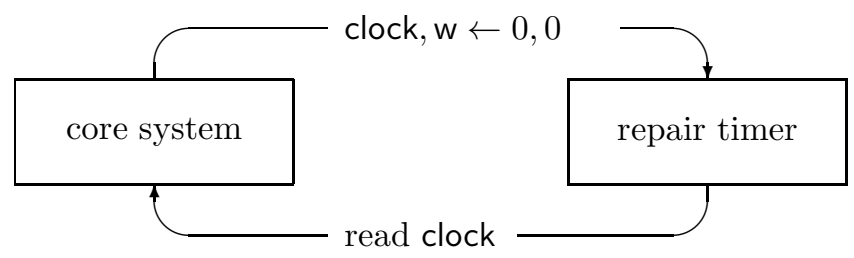

Figure 2: interface between core system and repair timer 
So that results from Section 4 are applicable to the composite system, each process invokes the repair timer (statements S2-S6) once in each cycle. Figure 1 includes S1 and S7 to present the repair timer in isolation, however in the context of a system invoking the repair timer, these two statements would be subsumed by statements reading registers at the beginning of a process cycle and writing registers at the end of a cycle.

A process configuration can be faulty with respect to the repair timer elements, the core system elements, or a combination of both elements. If a state $\sigma$ 's core projection violates $\mathcal{L}_{C}$ then $\sigma$ is said to be core-faulty; if $\sigma$ 's timer projection violates $\mathcal{L}_{T}$ then $\sigma$ is timer-faulty. While Definition 1 provides the basis for a precise characterization of a faulty repair timer, the situation for a general system can be ambiguous, as observed in Section 2.

Requirement 2 If $p$ 's process configuration is not core-faulty and Register ${ }_{q p}$ is faulty at $\sigma$, then the presence of a fault at $\sigma$ can be detected from the variables of $p$ and the contents of Register $_{q p}$.

In many cases it is not difficult to design a system satisfying Requirement 2, in spite of the ambiguity of a faulty process configuration - the requirement only specifies that $p$ detect the presence of a fault, and $p$ is not required to determine the fault's location (fault identification remains ambiguous). Depending on the particular computation, $p$ may not detect a fault. For instance, $q$ may repair its configuration, changing the contents of Register $_{p q}$, before $p$ reads the register.

The importance of Requirement 2 is that nonfaulty $p$ has the capability to detect a fault, retain the current values of its output variables, and initiate repair procedures. Moreover, $p$ can "contain" the fault because it reacts before copying values from Register ${ }_{p q}$ and transmitting them to other processes.

Requirement 3 Each cycle of a process invokes the repair timer. If, after reading registers at the start of a cycle, a fault can be inferred (as described in Requirement 2) for process $p$, and if $($ clock $>\mathcal{T}-\mathcal{D}$ ), then $p$ executes a double-reset. No other statements of the core system change the clock or w variables; any number of statements of the core system may read the clock variable. The legitimacy predicate for the core system does not depend on the clock or w variables of the repair timer.

Requirement 4 If any process $p$ executes a double-reset resulting in a state $\sigma$, then within $\mathcal{T}-7 \mathcal{D}$ rounds following $\sigma$, the core system component of the state is legitimate.

Requirement 4 means, for most core systems, that the core system stabilization time $\mathcal{M}$ satisfies $\mathcal{M} \leq \mathcal{T}-7 \mathcal{D}$. In essence, this is a constraint on $\mathcal{T}$, which is added to the constraint $\mathcal{T} \geq 11 \mathcal{D}$ given in Section 3 .

Lemma 16 If the core system is self-stabilizing with stabilization time $\mathcal{M}$ and satisfies Requirements 1 - 4 , then the system is self-stabilizing with stabilization time $\mathcal{M}+O(\mathcal{T})$, and the double-reset assignment executes at most once for each process in any computation.

The proof of Lemma 16 rests on the independence of the core system and the repair timer, as specified by Requirement 3, and the fact that the core system stabilizes before there is any possibility of executing a second double-reset by any process. The requirements do not, however, preclude the design of the core system from depending on repair timer properties. For instance, proving stabilization time $\mathcal{M}$ for the core system may depend on timer accuracy, since the core system can read clock variables 
during convergence to $\mathcal{L}_{C}$, and timer accuracy can be used in some circumstances to measure the progress of distributed algorithms and to allow processes to wait for such algorithms to stabilize.

More interesting than using the repair timer for stabilization is the use of the repair timer to enable time adaptive repair of output variables. The remainder of this section illustrates the use of the repair timer in two designs. Design 11 is a time adaptive system, repairing output variables in $O(\min (k, \mathcal{D}))$ rounds from any $k$-faulty initial state. The design requires that the core system use a sequence of repair procedures, following an idea developed in [8]. Output variables of nonfaulty processes may change to illegitimate values during convergence, but all output variables satisfy $\mathcal{L}_{O}$ within $O(\min (k, \mathcal{D}))$ rounds and continue to satisfy $\mathcal{L}_{O}$ thereafter. Design 2 is not fully time adaptive, but illustrates another use of the repair timer: the system can repair output variables in $O(r)$ rounds from any $k$-faulty initial state, $k \leq r$, and no nonfaulty process changes an output variable during repair.

Design 1 The core system has $\mathcal{D}$ independent repair procedures, denoted repair ${ }^{i}$ for $1 \leq i \leq \mathcal{D}$. Each of the repair procedures uses its own set of variables, including variables that are intended to be copied to the core system's output variables. Let output ${ }^{i}$ denote the set of variables of repair ${ }^{i}$ that correspond to the system's output variables, and let repair ${ }_{p}^{i}$ denote process $p$ 's portion of repair ${ }^{i}$. We suppose that the core system also prepares a set of variables output ${ }_{C}$ intended to be copied to output variables. Each repair procedure is invoked in every process cycle and repair is self-stabilizing to a predicate $\mathcal{L}^{i}$ within $\mathcal{M}$ rounds. When $\mathcal{L}_{C}$ holds, the output ${ }^{i}$ variables are equal to the system's output variables, for $1 \leq i \leq \mathcal{D}$, and output ${ }^{C}$ is also equal to the system's output.

Procedure repair ${ }^{i}$ has the property that, if the initial state is $j$-faulty, for $j \leq i$, then for all $p$, within $h \cdot i$ rounds, there occurs a state $\sigma$ such that for all $p$, the variables of output satisfy $\mathcal{L}_{O}$ (modulo renaming or copying their values to the system outputs) at $\sigma$ and all subsequent states. To exploit the repair timer, we suppose a stronger convergence property for repair ${ }^{i}$, namely that output ${ }_{p}^{i}$ variables stabilize within $h \cdot i$ of the $\mathrm{R}_{p}^{i}$-rounds.

Given any $k$-faulty initial state satisfying $k \leq \mathcal{D}$, certain repair procedures agree on values for output variables: for $i \geq k$, after repair ${ }^{i}$ stabilizes output ${ }^{i}$, any procedure repair ${ }^{\ell}$ for $\ell>i$ stabilizes output $^{\ell}$ to the same values that output ${ }^{i}$ has. Moreover, the core system stabilizes output ${ }^{C}$ to the same values contained in the stabilized output ${ }^{k}$ variables. The stabilized values of output sets are also constrained by distance from a fault: for any nonfaulty $p$ such that the minimum distance from $p$ to a faulty process is $d$, where $d>k$, then all of the output $p$ sets stabilize to the same values already contained in $p$ 's output variables.

The output ${ }_{p}$ sets are copied to the output variables of process $p$ as follows. In each cycle, if clock $p=\mathcal{T}$, then $p$ copies output ${ }_{p}^{C}$ to its output variables. Otherwise, in each cycle, $p$ copies output ${ }_{p}^{i}$ to its output variables where $i$ is the largest value satisfying $1 \leq i \leq \mathcal{D}$ and $(h+5) \cdot i \leq \operatorname{clock}_{p}$. No output set is copied to the output variables if $(h+5) \cdot \mathcal{D}<$ clock $_{p}<\mathcal{T}$ holds.

Theorem 4 If a system for Design 1 satisfies Requirements 1 A and $\mathcal{M}=O(\mathcal{D})$, then within $O(\min (k, \mathcal{D}))$ rounds following any $k$-faulty initial state, the system output-stabilizes to $\mathcal{L}_{O}$.

Proof: Consider a $k$-faulty initial state. If $k \geq \mathcal{D}$, then from $\mathcal{M}=O(\mathcal{D})$ and Theorem $\mathbb{1}$, the system stabilizes to $\mathcal{L}$ and hence $\mathcal{L}_{O}$ in $O(\mathcal{D})$ rounds, which proves the conclusion. The remaining case is $k<\mathcal{D}$ for a $k$-faulty initial state. For this case, we first show that any faulty process clock is time accurate within the first $k$ rounds. Requirement 3 ensures that some process within distance $k$ from any faulty process executes a double-reset in the first round, and Theorem 2 implies subsequent time accuracy within $k$ rounds. The same argument implies that each nonfaulty process within distance $k$ 
from a faulty process has a time-accurate clock after at most $k$ rounds. All nonfaulty processes have time-accurate clock variables throughout the computation.

Design 1 specifies that some nonfaulty processes do not change their output variables by any repair procedure, so the proof obligation is to show that faulty processes and those nonfaulty processes within distance $k$ to a faulty process stabilize their output variables in $O(k)$ time. After $k$ rounds, all such processes have time-accurate clock variables. By definition of time accuracy for a $k$-faulty initial state, a time-accurate clock ${ }_{p}$ variable with value $t$ implies that the number of $\mathrm{R}_{p}^{k}$-rounds preceding in the computation is at least $t-5 k$. Procedure repair ${ }^{k}$ converges within $h \cdot k$ of the $\mathrm{R}_{p}^{k}$-rounds, so after time accuracy holds, a clock ${ }_{p}$ value of $h \cdot k+5 k=(h+5) \cdot k$ implies that variables of output ${ }_{p}^{k}$ can be copied to $p$ 's output variables. The conditions of Design 1 also justify copying output ${ }_{p}^{j}$ to the output variables when clock $_{p} \geq(h+5) \cdot j$ for $k<j<\mathcal{D}$.

Having established the safety of copying output sets to the output variables, the remaining obligation is to show that all such copying either completes within $O(k)$ rounds or that any subsequent copying will not affect $\mathcal{L}_{O}$. Theorem 3 implies that all processes within distance $k$ to a faulty process will, after time accuracy holds, increase their clock variables to $(h+5) \cdot k$ within $O(k)$ rounds and will not subsequently decrease their clock values below this value. Therefore, within $O(k)$ rounds, all processes within distance $k$ to a faulty process assign their output variables, while those processes further than distance $k$ from a fault do not assign their output variables to falsify $\mathcal{L}_{O}$ by any step of the computation.

The repair ${ }^{i}$ procedures of Design 1 are independent, meaning that they do not share any of the variables they modify. Because the variables of repair ${ }^{C}$ are inactive for nonfaulty processes during the period of stabilization, they are a resource for faulty processes: values from nonfaulty output ${ }_{p}^{C}$ can be disseminated to other processes and used for the stabilization of repair ${ }^{i}$ procedures. For details on this technique, illustrated in a synchronous computation model, the reader is referred to [8].

Design 2 The core system uses procedure repair ${ }^{r}$ with a set of variables denoted output ${ }^{r}$ that are equal to the system output variables at a legitimate state. Procedure repair ${ }^{r}$ stabilizes the output $^{r}$ variables to satisfy $\mathcal{L}_{O}$ within $h \cdot r$ time from any initial state that is $j$-faulty for $j \leq r$; each faulty process $p$ stabilizes output ${ }_{p}^{r}$ after at most $h \cdot r$ of the $\mathrm{R}_{p}^{r}$-rounds occur. The output ${ }_{p}^{r}$ sets are copied to output variables of process $p$ as follows. In each cycle, if clock $p \geq(h+5) \cdot r$, then $p$ copies output ${ }_{p}^{r}$ to its output variables; for all other values of clock $p$ process $p$ leaves its output variables unchanged. The repair ${ }^{r}$ procedure stabilizes output ${ }_{p}^{r}$ to values already contained in $p$ 's output variables for any nonfaulty $p$.

Theorem 5 If a system for Design 2 satisfies Requirements 1 3, then within $O(r)$ rounds following any $k$-faulty initial state for $k \leq r$, the system output-stabilizes to $\mathcal{L}_{O}$. No step modifies output variables of nonfaulty processes to values differing from those specified by $\mathcal{L}_{O}$.

Theorem 5 can be verified by reasoning similar to the proof of Theorem 4 . Design 2 is not selfstabilizing and Theorem 5 does not specify Requirement 4 as a condition. The fault tolerance of this design is limited to $r$ faulty processes.

\section{Concluding Remarks}

It is challenging to construct a system that can repair variables inflicted by transient faults. A reasonable methodology for such system construction is based on tools for fault detection and repair, 
and these tools must themselves satisfy properties of time adaptivity and stabilization. This paper presented a phase clock algorithm specialized for the task of fault repair. The designs presented in Section 5 show how the repair timer can be composed with other system components.

Although time adaptivity and self-stabilization are major themes for this paper, the repair timer can be useful even when neither full stabilization nor fast stabilization is needed, because it is convenient to reason about the progress of repair procedures by measuring elapsed time (which would otherwise be complicated due to possible corruption of time-measurement variables). An observer of the system located at process $p$ could monitor repair progress by repeatedly examining clock ${ }_{p}$, possibly delaying critical activity until repair is complete.

Use of the repair timer can add overhead to repair procedures because each cycle of repair invokes the timer, and the clock variable only increments in relation to rounds. It could be that actual repair only involves a small subset of processes, but a clock variable will not, in general, increment $t$ times unless all processes at distance $d$ have completed $t-d$ cycles - including processes that are not involved in the repair. Thus the measurement of repair time in rounds could be overly pessimistic and cause processes to wait longer than necessary before they infer that repair is complete. Another slowing of repair timing results if a loose upper bound on the network diameter is used for $\mathcal{D}$ (an upper bound is typically proposed for dynamic networks) since $\mathcal{T}$, the "resting value" for the repair timer, is determined by the value $\mathcal{D}$.

\section{References}

[1] Y Afek and S Dolev. Local stabilizer. In Proceedings of the 5th Israeli Symposium on Theory of Computing and Systems, pages 74-84, 1997.

[2] A Arora, S Dolev, and MG Gouda. Maintaining digital clocks in step. Parallel Processing Letters, 1:11-18, 1991.

[3] H Attiya and J Welch. Distributed Computing: Fundamentals, Simulations, and Advanced Topics. McGraw-Hill, London, 1998.

[4] B Awerbuch. Complexity of network synchronization. Journal of the ACM, 32:804-823, 1985.

[5] J Beauquier, C Genolini, and S Kutten. Optimal reactive k-stabilization: the case of mutual exclusion. In PODC99 Proceedings of the Nineteenth Annual ACM Symposium on Principles of Distributed Computing, pages 209-218, 1999.

[6] JM Couvreur, N Francez, and MG Gouda. Asynchronous unison. In ICDCS92 Proceedings of the 12th International Conference on Distributed Computing Systems, pages 486-493, 1992.

[7] S Dolev. Self-Stabilization. The MIT Press, Cambridge, Massachusetts, 2000.

[8] S Dolev and T Herman. Parallel composition of stabilizing algorithms. In WSS99 Proceedings of the 1999 ICDCS Workshop on Self-Stabilizing Systems, pages 25-32. IEEE Computer Society, 1999.

[9] S Ghosh and A Gupta. An exercise in fault-containment: self-stabilizing leader election. Information Processing Letters, 59:281-288, 1996.

[10] S Ghosh, A Gupta, T Herman, and SV Pemmaraju. Fault-containing self-stabilizing algorithms. In PODC96 Proceedings of the Fifteenth Annual ACM Symposium on Principles of Distributed Computing, pages 45-54, 1996. 
[11] S Ghosh, A Gupta, and SV Pemmaraju. A fault-containing self-stabilizing algorithm for spanning trees. Journal of Computing and Information, 2:322-338, 1996.

[12] MG Gouda and T Herman. Stabilizing unison. Information Processing Letters, 35:171-175, 1990.

[13] T Herman. A stabilizing repair timer. In DISC98 Proceedings of the 12th International Symposium on Distributed Computing, LNCS 1499, pages 186-200, 1998.

[14] S Kutten and B Patt-Shamir. Time-adaptive self stabilization. In PODC97 Proceedings of the Sixteenth Annual ACM Symposium on Principles of Distributed Computing, pages 149-158, 1997.

[15] S Kutten and B Patt-Shamir. Asynchronous time-adaptive self stabilization. In PODC98 Proceedings of the Seventeenth Annual ACM Symposium on Principles of Distributed Computing, page 319, 1998.

[16] L Lamport. Time, clocks, and the ordering of events in a distributed system. Communications of the ACM, 21:558-564, 1978.

[17] F Mattern. Virtual time and global states of distributed systems. In Parallel and Distributed Systems, pages 215-226. Elsevier Science Publishers, 1989. M Cosnard et al (eds).

\section{Appendix: Proofs}

Proof of Lemma 1: The essence of the proof is that neither S3 nor S6 increment a clock to a value two greater than any neighbor. Since Definition 2 involves $x$ variables, the effect of statement S1 requires examination. Reading a register to assign an $x$ variable only increases the accuracy of the image variable; in particular, given $\left(b_{p q} \wedge b_{q p}\right)$ as a precondition, S1 does not falsify this condition, because $p$ and $q$ have clock values differing by at most one in the precondition. Therefore it suffices to verify that any change to clock ${ }_{p}$ or clock $_{q}$ also satisfies the lemma. In a reset-free computation, only S3 and S6 change a clock variable. If S3 executes, incrementing clock ${ }_{p}$, we have clock $\mathbf{k}_{p} \leq \mathrm{x}_{p}[q]$ as a precondition. Since $b_{p q}$, we have $\operatorname{clock}_{p} \in\left\{\operatorname{clock}_{q}\right.$, lock $\left._{q}+1\right\}$ also as a precondition; thus the increment to clock $p$ results in a state satisfying $\mid$ clock $_{p}-$ clock $_{q} \mid<2$, verifying $b_{p q}$. The postcondition also satisfies $b_{q p}$, since the change to clock $\mathrm{k}_{p}$ does not alter the relation between $\operatorname{clock}_{q}$ and $\mathrm{x}_{q}[p]$. A similar argument applies to $\mathbf{S 6}$, and also to the case of $q$ incrementing its clock.

Proof of Lemma 2: By definition of a rising computation, each process has a lower bound on neighboring clock variables in its $\mathrm{x}$ variable, because clock values cannot decrease in a reset-free computation. Suppose $p$ is the first of $(p, q)$ to increment its clock. A precondition for this step is clock $\leq_{p} \mathrm{x}_{p}[q]$, which implies clock $p \leq \operatorname{clock}_{q}$, which in turn implies $\mathrm{x}_{q}[p] \leq \operatorname{clock}_{q}$. Consider two cases for this last inequality, $(i) \mathrm{x}_{q}[p]<\operatorname{clock}_{q}$ or $(i i) \mathrm{x}_{q}[p]=\operatorname{clock}_{q}$. For $(i)$, process $q$ cannot increment clock $_{q}$, and this situation will persist until $p$ increments its clock sufficiently many times so that clock $p \geq$ clock $_{q}$. It is straightforward to verify that $p$ does not increment clock beyond clock $_{q}+1$, so for case $(i)$ the first increment to clock $_{q}$ establishes $\left(b_{p q} \wedge b_{q p}\right)$. For $(i i)$, we deduce from the inequalities above (clock $_{q} \leq$ clock $_{p} \wedge$ clock $_{p} \leq$ clock $_{q}$ ) holds as precondition to $p$ 's first increment step, and the inequalities with regard to the $\mathrm{x}$ variables are similar. So for case $(i i),\left(b_{p q} \wedge b_{q p}\right)$ holds directly.

Proof of Lemma 3: By Lemma 2, neighboring $(p, q)$ establish $\left(b_{p q} \wedge b_{q p}\right)$ at or before $\sigma$; by Lemma 1 such processes continue to satisfy this property for the remainder of the reset-free computation segment. 
Proof of Lemma 4: Because we consider a based computation, and not a rising computation in this lemma, the invariance of $\left(b_{p q} \wedge b_{q p}\right)$ stated in Lemma 1 is not applicable. Note that $\left(\forall p:: \neg g a p_{p}\right)$ holds at a smooth state. The invariance of smoothness is therefore verified from the conditions of S3-S6, since no gap exists at a smooth state and S3 preserves smoothness. It is also simple to verify that the least clock value, if smaller than $\mathcal{T}$, increments within two rounds from a smooth state, hence at most $2 \mathcal{T}=O(\mathcal{D})$ rounds are needed to obtain a state satisfying $\left(\forall p::\right.$ clock $\left._{p}=\mathcal{T}\right)$. A similar argument shows that all w variables converge to $3 \mathcal{D}+1$ within $O(\mathcal{D})$ rounds.

Proof of Lemma A: In the first round, $p$ reads neighboring clock values and detects local minimality. If $p$ increments in this round, the lemma holds; and if $p$ does not increment, it writes its clock and

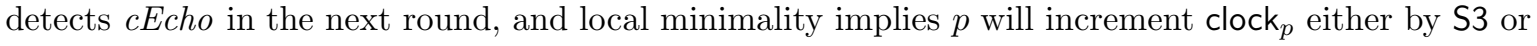
S6.

Proof of Lemma 6: Observe that $\left(\forall p:: \mathrm{w}_{p}=3 \mathcal{D}+1\right)$ holds at least until some clock exceeds $\mathcal{T}-\mathcal{D}$ so that $\mathrm{S} 4$ can execute. And $\mathrm{w}_{p}=3 \mathcal{D}+1 \Rightarrow w B i g_{p}$, so process $p$ does not execute the assignment of S5. This implies that the computation is reset-free until some clock obtains the value exceeding $\mathcal{T}-\mathcal{D}$. Lemma 5 implies that each minimal clock value increments in any pair of rounds, which implies that the maximum of the set of clock values eventually grows as the computation proceeds. Let clock be the first clock to attain the value $8 \mathcal{D}+1$ at state $\alpha$. Thus $\left(\forall q: q \in \mathcal{N}_{p}\right.$ : $\left.\operatorname{clock}_{q} \geq 8 \mathcal{D}\right)$ holds prior to $\alpha$. More generally, it follows by induction that $\left(\forall q: \operatorname{dist}_{q p}=k>0: \operatorname{clock}_{q} \geq 8 \mathcal{D}-k\right)$. Therefore, each clock value has incremented at least once prior to $\alpha$. Let clock $_{q}$ be the first clock to attain the value $9 \mathcal{D}+1$ at state $\beta$. At state $\beta$, each process has incremented its clock twice in a reset-free computation, implying that each process has read all of its registers at least once in this reset-free computation. Therefore the computation segment beginning with $\beta$ is by definition a rising computation segment (at least until some clock exceeds $\mathcal{T}-\mathcal{D}$ ). Now let clock $_{r}$ be the first clock to attain the value $10 \mathcal{D}+1$ at state $\gamma$. At state $\gamma$, each process has incremented its clock at least once in a rising computation, and by Lemma $3, \gamma$ is a smooth state.

Proof of Lemma 7: Lemma 6 shows that the computation contains a smooth state, so the obligation here is to show the $O(\mathcal{D})$ time bound. By Lemma 5 each minimal clock value increments at least once in any two consecutive rounds, so within $20 \mathcal{D}+2$ rounds, some clock attains the value $10 \mathcal{D}+1$, establishing a smooth state.

Proof of Lemma 8: The proof begins with a claim on the first $k$ rounds of the based computation: within $k$ rounds there is a state satisfying

$$
\begin{aligned}
& \left(\forall q: \operatorname{dist}_{q r}=k: \mathrm{w}_{q} \leq 2 k \wedge \operatorname{clock}_{q} \leq 2 k\right) \\
& \left(\forall q, j: j<k \wedge \text { dist }_{q r}=j: \mathrm{w}_{q} \leq 3 k-j \wedge \operatorname{clock}_{q} \leq 3 k-j\right)
\end{aligned}
$$

The claim is shown by induction. The first state of the computation satisfies the claim for $k=0$ as the base case. Suppose the claim holds for $k \leq \ell$ and consider two processes $q$ and $s$ such that dist $t_{r q}=\ell$, $s \in \mathcal{N}_{q}$, and distrs $=\ell+1$. Let $\sigma$ be a state satisfying (1)-(2) for $k=\ell$. By the $c E c h o$ condition of S3, process $q$ does not increment clock $_{q}$ beyond $2 \ell$ until process $s$ 's image fields in Register ${ }_{s q}$ have the appropriate values. In fact, these register fields may initially have the appropriate values, which would allow $q$ to increment clock and $w$ variables to $2 \ell+1$ by S2-S3. However process $q$ cannot subsequently increment to $2 \ell+2$ until the $c E c h o$ condition holds, which requires a cycle by $s$ (and all other neighbors). Process $s$ therefore observes $y_{s}[q] \leq 2 \ell+1$ in its cycle and assigns at most $2 \ell+2$ to its $w$ and clock variables. Since $\sigma$ occurs at least by round $\ell$, the bound of $2 \ell+2$ for $s$ variables applies within round $\ell+1$, which establishes (1) of the claim. 
Condition (2) is also shown by induction. For $k=0$, the base case, (2) holds vacuously. Now suppose (2) holds for $k \leq \ell$ and consider two processes $q$ and $s$ such that dist $t_{r q}=\ell, s \in \mathcal{N}_{q}$, and dist $t_{r s}=\ell+1$. Condition (1) places an upper bound on variables at distance $\ell+1$ from process $r$ within round $\ell+1$. Therefore clock $_{s} \leq 2(\ell+1)$ within round $\ell+1$. In moving from round $\ell$ to $\ell+1$, we consider the

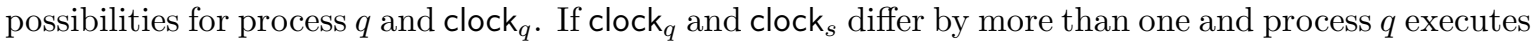

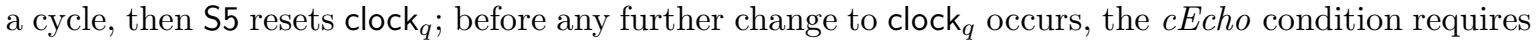
a full cycle by $s$, which validates (2) up to distance $\ell+1$ within round $\ell+1$. If clock $_{q}$ and clock are equal or differ by one, then clock $_{q}$ could increment. Observe here that no clock or w variable can increment beyond one more than any neighboring value; by another inductive argument, no clock or $\mathrm{w}$ variable increments beyond $j$ more than any corresponding variable at distance $j$. Therefore clock ${ }_{q}$ does not increment beyond $(2 \ell+2)+1$ so long as clock $\leq 2 \ell+2$. This observation is generalized by (2) for $k=\ell+1$ within round $\ell+1$. Note that we have assumed that any clock increment is due to $\mathrm{S} 3$ and not $\mathrm{S} 6$ in this argument; this assumption is justified by (1), since $\mathrm{w}<3 \mathcal{D}+1$, which disables execution of S6.

Proof of Lemma 9: Let $\sigma$ be a state satisfying $\left(\forall q::\right.$ clock $\left._{q} \leq 3 \mathcal{D}\right)$. By Lemma 8 such a state $\sigma$ occurs with $\mathcal{D}$ rounds of the based computation. So long as every clock is at most $\mathcal{T}-\mathcal{D}$, no step subsequent to $\sigma$ decreases a $\mathrm{w}$ variable; and if no $\mathrm{w}$ variable is reset by $\mathrm{S} 4$ in a consecutive pair of rounds, then the minimum value of the set of $w$ variables either increases by that pair of rounds or all $\mathrm{w}$ variables already have the maximum $3 \mathcal{D}+1$ value (we consider a consecutive pair of rounds to ensure that $w E$ cho will hold for $\mathrm{S} 2)$. Therefore, if no clock variable attains the value $7 \mathcal{D}+1$ within $2 \cdot(3 \mathcal{D}+1)$ rounds, all $w$ variables equal $3 \mathcal{D}+1$ and the lemma holds. On the other hand, if some clock does attain the value $7 \mathcal{D}+1$, we shall deduce that all $w$ values equal $3 \mathcal{D}+1$, which also proves the lemma. The argument rests on the following claim: at all states subsequent to $\sigma$ satisfying ( $\forall p::$ clock $\left._{p} \leq 7 \mathcal{D}\right)$, the implication clock $p \geq 3 \mathcal{D}+k \Rightarrow \mathrm{w}_{p} \geq k$ holds for every $p$ and $0 \leq k \leq 3 \mathcal{D}+1$. This claim is verified by induction on $k$. For $k=0$ the result is immediate from the domain of $\mathrm{w}$ variables. Now consider $k>0$ and suppose the claim holds for $k-1$. Let $q$ be the first process to assign clock $_{q} \leftarrow 3 \mathcal{D}+k$. If the assignment occurs by $\mathrm{S} 6$ then $w=3 \mathcal{D}+1$ and the claim holds; if the assignment occurs by $\mathrm{S} 3$, then each neighbor of $q$ has a clock value of $3 \mathcal{D}+(k-1)$, hence by hypothesis each neighboring $\mathrm{w}$ variable is at least $k-1$, and $\mathrm{w}_{q} \geq k-1$ by the same hypothesis. The result is that the same cycle assigning clock $\mathrm{k}_{q} \leftarrow 3 \mathcal{D}+k$ also assigns $\mathrm{w}_{q}$ to be at least $k$. Similar arguments treat the general case for $q$ (not necessarily the first) assigning $3 \mathcal{D}+k$ to clock $_{q}$, verifying that $\mathrm{w}_{q} \geq k$ as a result. To complete the lemma, consider the first state $\delta$ where some clock $_{q}$ has value $7 \mathcal{D}+1$. By the induction argument given in the proof of Lemma 8, any clock at distance $j$ from clock ${ }_{q}$ has had a value of at least $7 \mathcal{D}-j$ prior to state $\delta$. Therefore every clock has contained a value of at least $6 \mathcal{D}+1$ prior to $\delta$, implying that each $\mathrm{w}$ variable is at least $3 \mathcal{D}+1$ prior to $\delta$. The state immediately preceding $\delta$ thus satisfies proof obligation.

Proof of Lemma 11: by induction on $t$. For $t=0$ let $\sigma^{\prime}=\sigma$ to satisfy the base case. For $t>0$, we have clock $q \leq 3 t \wedge \mathrm{w}_{q} \leq 3 t$ by hypothesis. By the Echo conditions of S2, S3 and S6, the clock and $\mathrm{w}$ values of $q$ remain at most $3 t$ until all neighbors either $(i)$ complete cycles that observe these values and write corresponding images to output registers or (ii) happen to have these values already in their output registers.

Considering $(i)$, for $r \in \mathcal{N}_{q}$ satisfying dist $t_{p r}=t+1$, the execution of S2 assures $\mathrm{w}_{r} \leq 3 t+1$ within one round, and clock ${ }_{r}$ is at most $3 t+1$ if $r$ observes no gap, or assigned some value at most $w_{r}$ otherwise; either case verifies the inductive hypothesis for $t+1$. These considerations for $(i)$ also verify the second part of the lemma, which concerns a path of unperturbed processes, and the same hypothesis with $3 t$ replaced by $t$. 
Considering (ii), process $q$ may increment clock $_{q}$ and $\mathrm{w}_{q}$ because $r \in \mathcal{N}_{q}$ happens already to have values corresponding to clock $q$ and $\mathrm{w}_{q}$ in its output register fields. In this case, $q$ may increment its variables to at most $3 t+1$ immediately. Furthermore process $r$ may initially have its program counter at S7, about to write its image variables in such a way that $q$ can observe the $c E c h o$ condition (even though $r$ would not actually read and write in a full cycle). Therefore, if $r$ executes S7, process $q$ can increment variables again to at most $3 t+2$. However, here a $c E c h o$ condition will not be satisfied at $q$ until all neighbors complete full cycles, so $q$ 's variables cannot exceed $3 t+2$ until $r$ completes a cycle. When $r$ does complete a cycle, by the reasoning above for $(i)$ we deduce that clock $_{r} \leq 3 t+3$ and $\mathrm{w}_{r} \leq 3 t+3$ for $r \in \mathcal{N}_{q}$.

Proof of Lemma 12: Note that the lemma holds trivially if the initial state is $n$-perturbed. For the case $k<n$ we use induction on $t$ and nested induction on $k$ and suppose a based computation. For the base case $t=0$ consider $p \in \mathcal{N}_{q}$. Since $q$ is perturbed, there is a path $P$ from $p$ to some perturbed $r$ (possibly through $q$ ) of $k+2$ or fewer processes, which is not smooth. Because clock $p=\mathcal{T}$, some neighboring pair of processes along path $P$ has the property that one clock exceeds $\mathcal{T}-\mathcal{D}$ while the other is less than $\mathcal{T}-\mathcal{D}$. Therefore some process in path $P$ executes $\mathrm{S} 4$ in the first round. By the arguments of Lemma 11 it follows that $p$ executes $\mathbf{S} 4$ within $k+2$ rounds. This completes the base case, but reasoning similar to the nested induction also applies for $t>0$. Finally, because the initial state may not justify a based computation, two additional rounds are added to conclude a $k+t+4$ bound.

Proof of Lemma 13: Lemma 10 states that a process executes S4 at most once in a computation, so it suffices to show that $p$ either does not execute S4 or executes S4 within the first $4+\min (\mathcal{D}, t+k)$ rounds. If $p$ is unperturbed, Lemma 12 implies the result. If $p$ is perturbed, then for some perturbed region $P$ containing $p$, there is an unperturbed $q$ neighboring some process of $P$ that executes $\mathrm{S} 4$ within the first $4+\min (\mathcal{D}, k)$ rounds by Lemma 12. Applying Lemma 11 we deduce that $\operatorname{clock}_{p} \leq 3 \min (\mathcal{D}, k)$ holds after $\min (\mathcal{D}, k)$ additional rounds, and by arguments of Lemmas 8 and 9 process $p$ does not execute S4 in the remainder of the computation. Therefore, for perturbed $p$, the distance from $p$ to a perturbed process is $t=0$ and after $4+\min (\mathcal{D}, k)+\min (\mathcal{D}, k)$ rounds, process $p$ does not execute S4.

Proof of Lemma 14: by induction on $t$. The base case $t=0$ trivially follows from the domain of $w$ variables, which have non-negative values. The same observation concerning the domain of $w$ variables simplifies the proof obligation to the case dist $t_{p q} \leq t$. It is useful also to observe base cases for $t=1$ and $t=2$, since by the end of round two the computation is based, which simplifies reasoning for higher rounds. For $t=1$ the verification is again trivial by the domain of $\mathrm{w}$ variables. For $t=2$, it is required to show that by the end of round two, $w_{p} \geq 1$. In fact any change to $w_{p}$ is an increase from its original value of zero, and at least one increment occurs because $w E c h o_{p}$ is observed by $p$ within two rounds following $\sigma$. No subsequent reduction to $\mathrm{w}_{p}$ results in a value less than one, since $w_{M} \operatorname{Tin}_{p}$ is at least zero at all states. This verifies the base case for $t=2$.

Now suppose the hypothesis $\mathrm{w}_{q} \geq\left\lfloor\left(t-\right.\right.$ dist $\left.\left._{p q}\right) / 2\right\rfloor$ for every $q$ such that dist $t_{p q} \leq t$ at some state $\sigma^{\prime}$. Note that no such process $q$ subsequently executes $S 4$ in the computation, by Lemma 11; therefore any subsequent change to $\mathrm{w}_{q}$ occurs by $\mathrm{S} 2$. If S2 assigns $\mathrm{w}_{q}$ a value at least $\left[\left((t+1)-\right.\right.$ dist $\left.\left._{p q}\right) / 2\right\rfloor$ in the round following $\sigma^{\prime}$, or if $\mathrm{w}_{q}$ already has such a value and does not decrease, then the induction step is verified. Therefore we consider the possibility that $\mathrm{w}_{q}$ either remains unchanged or decreases below $\left\lfloor\left(t-\right.\right.$ dist $\left.\left._{p q}\right) / 2\right\rfloor$ by execution of S2. A decrease only occurs if $\mathrm{w}_{q}>w \operatorname{Min}_{q}+1$, so a decrease below $\left\lfloor\left(t-\right.\right.$ dist $\left.\left._{p q}\right) / 2\right\rfloor$ is only possible if there is a neighbor $r \in \mathcal{N}_{q}$ satisfying $\mathrm{y}_{q}[r] \leq\left\lfloor\left(t-\right.\right.$ dist $\left.\left._{p q}\right) / 2\right\rfloor-2$, which would in turn imply that such a value existed in $\mathrm{w}_{r}$ in the previous round. But by hypothesis, 
$\mathrm{w}_{r} \geq\left\lfloor\left(t-\right.\right.$ dist $\left.\left._{p r}\right) / 2\right\rfloor$, and since $r \in \mathcal{N}_{q}$ the value of $\mathrm{w}_{r}$ is at least $\left\lfloor\left(t-\right.\right.$ dist $\left.\left._{p q} \pm 1\right) / 2\right\rfloor$, which contradicts $\mathrm{y}_{q}[r] \leq\left\lfloor\left(t-\right.\right.$ dist $\left.\left._{p q}\right) / 2\right\rfloor-2$. Therefore such a decrease to $\mathrm{w}_{q}$ cannot occur.

The remaining case to consider is that $\mathrm{w}_{q}=\left\lfloor\left(t-\right.\right.$ dist $\left.\left._{p q}\right) / 2\right\rfloor$ and does not change in the round following $\sigma^{\prime}$. Here there are two cases for $t$ and $q$, either $\left(t-d_{i s t_{p q}}\right)$ is even or it is odd. If $\left(t-d_{i s t}\right)$ is even, then $\left\lfloor\left(t-\right.\right.$ dist $\left.\left._{p q}\right) / 2\right\rfloor$ is equal to $\left\lfloor\left((t+1)-\right.\right.$ dist $\left.\left._{p q}\right) / 2\right\rfloor$ and the hypothesis for $(t+1)$ is proved - the value of $\mathrm{w}_{q}$ can remain unchanged in the round following $\sigma^{\prime}$ and satisfy the hypothesis. If, however, $\left(t-\right.$ dist $\left._{p q}\right)$ is odd, then $\mathrm{w}_{q}$ is required to increment to verify the hypothesis for $(t+1)$. Observe

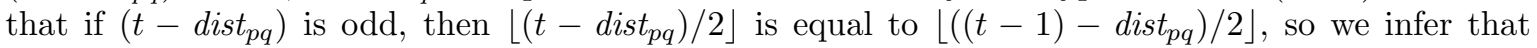
$\mathrm{w}_{q}=\left\lfloor\left(t-\right.\right.$ dist $\left.\left._{p q}\right) / 2\right\rfloor$ held at round $(t-1)$ (here we assume the hypothesis not only for $t$, but $(t-1)$ as well, which is permissible because base cases for $t=1$ and $t=2$ have been verified). Therefore by round $(t+1)$, process $q$ observes $w E c h o_{q}$ and increments $\mathrm{w}_{q}$, which verifies the hypothesis for $(t+1)$.

Proof of Lemma 15: by induction on $t$, for $t \geq 0$. Note that round $t+2$ occurs in a based computation, since within two rounds following $\sigma$ the computation is based. The base case for induction is shown for $t=0$ and $t=1$, since the main induction step relies on two previous rounds of a based computation. For $t \leq 1$, since every clock variable is at least zero, the base cases are verified directly by the domain of clock variables - which are at least zero at any state.

Note that for any $t, t-2 \leq$ dist $_{p q}$ trivially satisfies the conclusion because clock variables are always at least zero; therefore in the remainder of the proof we consider only the case of $q$ and $t$ satisfying

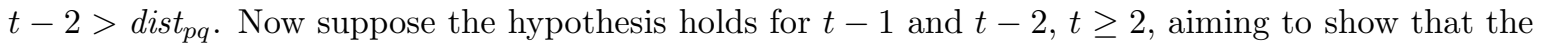
hypothesis also holds for $t$, that is, that clock variables satisfy the specified lower bound by the end of round $t+2$.

By Lemmas 11 and 12, by round $t$, any process in the set $R=\left\{r \mid\right.$ dist $\left._{p r} \leq t-3\right\}$. has either executed S4 or will not do so throughout the remainder of the computation. Therefore in round $t+2$, any reduction to clock ${ }_{r}$ for $r \in R$ could only occur by S5. Lemma 14 establishes that $\mathrm{w}_{r} \geq$ $\left\lfloor\left((t+1)-\right.\right.$ dist $\left.\left._{p r}\right) / 2\right\rfloor$ holds invariantly following round $t+1$. So if process $r$ executes $\mathrm{S} 5$, the result satisfies clock $\mathrm{c}_{r} \geq\left\lfloor\left((t-2)-\right.\right.$ dist $\left.\left._{p r}\right) / 2\right\rfloor$, which would verify the inductive hypothesis for $r$ and round $t+2$. If $r$ does not execute $\mathrm{S} 5$ in round $x+1$, then consider two cases for $r$.

Case: $t-$ dist $_{p r}$ is even. Observe that $\left\lfloor\left((t-2)-\right.\right.$ dist $\left.\left._{p r}\right) / 2\right\rfloor$ differs from $\left\lfloor\left((t-3)-\right.\right.$ dist $\left.\left._{p r}\right) / 2\right\rfloor$, meaning that the obligation is to show that clock ${ }_{r}$ is either at least $\left\lfloor\left((t-2)-\right.\right.$ dist $\left.\left._{p r}\right) / 2\right\rfloor$ by the end of round $t+1$, or that clock $\mathbf{k}_{r}$ increments during round $t+2$. If the former holds, the hypothesis is proved, so suppose clock $_{r}=\left\lfloor\left((t-3)-\right.\right.$ dist $\left.\left._{p r}\right) / 2\right\rfloor$ at the end of round $t+1$. Because $t-$ dist $_{p r}$ is even, clock $_{r} \geq\left\lfloor\left((t-4)-\right.\right.$ dist $\left.\left._{p r}\right) / 2\right\rfloor$ by hypothesis for $t-2$. But this implies that during round $t+1$, the value of clock ${ }_{r}$ either did not change or was reduced by S5. However a reduction by S5 would satisfy the hypothesis for $t$ as well, because of Lemma 14's bound on w variables. The only remaining possibility is that clock ${ }_{r}$ does not change in round $t+1$, implying that $r$ observes $c E c h o$ during round $t+2$. Therefore, if clock ${ }_{r} \leq c$ Min $_{r}$ when $r$ observes $c E c h o$, then clock ${ }_{r}$ will increment either by S3 or S6. To show that $r$ does indeed observe $c E c h o$, we use the hypothesis for $t-1$ and each $q \in \mathcal{N}_{r}$. If dist $_{p q} \leq$ dist $_{p r}$, then by round $t+1$ (and throughout round $t+2$ ) the relation clock $_{q} \geq$ clock $_{r}$ holds at least until $r$ increments its clock. If dist $_{p q}=$ dist $_{p r}+1$, then $\left\lfloor\left((t-2)-\right.\right.$ dist $\left.\left._{p r}\right) / 2\right\rfloor$ and $\left\lfloor\left((t-2)-\right.\right.$ dist $\left.\left._{p q}\right) / 2\right\rfloor$ are equal, and again the relation clock $_{q} \geq$ clock $_{r}$ holds at least until $r$ increments its clock.

Case: $t$-dist $t_{p r}$ is odd. A similar detailed argument can be given for this case, but there is a simpler approach: $\left\lfloor\left((t-2)-\right.\right.$ dist $\left.\left._{p r}\right) / 2\right\rfloor$ and $\left\lfloor\left((t-3)-\right.\right.$ dist $\left.\left._{p r}\right) / 2\right\rfloor$ are equal, so the hypothesis for $t-1$ and $r$ directly suffice to verify the hypothesis for $t$. 
Proof of Lemma 16: In any computation, either some process executes a double-reset or no process does so. In the latter case, the core system component stabilizes within $\mathcal{M}$ rounds, and the repair timer concurrently reaches the timer-final condition within $O(\mathcal{T})$ rounds by Theorem 11. This demonstrates $\mathcal{M}+O(\mathcal{T})$ stabilization time if no double-reset occurs; the same argument applies to the case where any double-reset occurs by S4 and not by the core system. Lemma 10 implies that a double-reset occurs at most once for each process in this case.

Now consider the possibility that the core system executes a double-reset at least once in a computation. All such assignments cease after the base system stabilizes, which occurs within $\mathcal{M}$ rounds, so the system stabilization time is $\mathcal{M}+O(\mathcal{T})$. To show that any process executes a double-reset at most once, we demonstrate that the core system stabilizes before clock $>\mathcal{T}-\mathcal{D}$ holds at any process, since Requirement 3 prevents repeated resets of the clock so long as clock $\leq \mathcal{T}-\mathcal{D}$.

If any double-reset assignment occurs, then within $\mathcal{D}$ rounds thereafter, a state $\sigma$ occurs such that each clock is at most $3 \mathcal{D}$ by Lemma 11, and also within $\mathcal{D}$ rounds, time-accuracy holds and is invariant thereafter by Theorem 2. Although Theorem 2 is conditioned on $k<n$ for a $k$-perturbed initial state, its proof arguments are valid for the case of an $n$-faulty initial state, provided some process executes a double-reset in the first round. While we do not suppose that a double-reset occurs in the first round, the state preceding the first double-reset can be considered as the initial state for the subsequent computation, so that Theorem 2/'s results apply for the suffix computation. Time accuracy for the extreme case of an $n$-faulty initial state implies for clock $=t$ that at least $(t-\mathcal{D}-5 \mathcal{D})=t-6 \mathcal{D}$ rounds have transpired. Therefore, if $\mathcal{T}-\mathcal{D} \geq X+6 \mathcal{D}$, where $X$ is the number of rounds needed for stabilization, then as soon as time accuracy holds, no clock increases beyond $\mathcal{T}-\mathcal{D}$ until the core system has stabilized. Requirement $\mathbb{\theta}$ implies stabilization within $\mathcal{T}-7 \mathcal{D}$ rounds, which ensures that the core system stabilizes before there is the possibility of a second double-reset. To complete the proof we address the period between the first double-reset and before time accuracy holds. This is at most $\mathcal{D}$ rounds, and it is easy to show that no clock increases from zero to beyond $\mathcal{T}-\mathcal{D}$ within $\mathcal{D}$ rounds, so a second double-reset does not occur in the period before time accuracy holds. 